\title{
Video Article \\ Culture, Manipulation, and Orthotopic Transplantation of Mouse Bladder Tumor Organoids
}

\author{
Yubin Kim** ${ }^{1}$, Juhee Lee* ${ }^{1}$, SungEun Kim* ${ }^{1}$, Kunyoo Shin ${ }^{1}$ \\ ${ }^{1}$ Department of Life Sciences, Pohang University of Science and Technology \\ * These authors contributed equally
}

Correspondence to: Kunyoo Shin at kunyoos@postech.ac.kr

URL: https://www.jove.com/video/60469

DOI: doi:10.3791/60469

Keywords: tumor organoids, 3D culture, bladder tumor, invasive urothelial carcinoma, lentiviral transduction, orthotopic transplantation

Date Published: $1 / 23 / 2020$

Citation: Kim, Y., Lee, J., Kim, S., Shin, K. Culture, Manipulation, and Orthotopic Transplantation of Mouse Bladder Tumor Organoids. J. Vis. Exp. (), e60469, doi:10.3791/60469 (2020).

\section{Abstract}

The development of advanced tumor models has long been encouraged because current cancer models have shown limitations such as lack of three-dimensional (3D) tumor architecture and low relevance to human cancer. Researchers have recently developed a 3D in vitro cancer model referred to as tumor organoids that can mimic the characteristics of a native tumor in a culture dish. Here, experimental procedures are described in detail for the establishment of bladder tumor organoids from a carcinogen-induced murine bladder tumor, including culture, passage, and maintenance of the resulting 3D tumor organoids in vitro. In addition, protocols to manipulate the established bladder tumor organoid lines for genetic engineering using lentivirus-mediated transduction are described, including optimized conditions for the efficient introduction of new genetic elements into tumor organoids. Finally, the procedure for orthotopic transplantation of bladder tumor organoids into the wall of the murine bladder for further analysis is laid out. The methods described in this article can facilitate the establishment of an in vitro model for bladder cancer for the development of better therapeutic options.

\section{Video Link}

The video component of this article can be found at https://www.jove.com/video/60469/

\section{Introduction}

Bladder cancer is the most prevalent urinary tract cancer, with approximately 165,000 patients dying annually ${ }^{1}$. Among the various types of bladder cancer, muscle-invasive urothelial carcinoma exhibits an aggressive phenotype, and its 5 year survival rate is lower than $50 \%{ }^{2}$. Novel therapeutic options for invasive urothelial tumors have not been expanded over the last few decades ${ }^{1}$.

Cancer cell lines have been extensively used for drug screening ${ }^{3}$. Although favorable results have been observed in numerous drug candidates in cancer cell lines, poor results are reported in clinical trials ${ }^{4}$. Following increased adaptation to in vitro two-dimensional (2D) culture environments, it has become increasingly difficult to recapitulate native tumors in cell lines. Animal cancer models or patient-derived tumor xenografts can be used to address the limitations observed in bladder cancer cell lines. However, animal cancer models are time and resource intensive. Therefore, improved disease models have been on demand for years and a novel model system, organoids, has been developed to overcome the shortcomings of existing models ${ }^{5}$.

An organoid is a multicellular 3D construct that can recapitulate in vitro the physiological characteristics of its corresponding in vivo organ. Normal and tumor organoids can be derived from either pluripotent or adult stem cells, and primary tumor cells, respectively,6. Over the last several years, tumor organoids have been established from a large number of diverse tumor tissues ${ }^{7}$, including colon ${ }^{8,9}$, bladder ${ }^{10}$, pancreas $^{11,12}$, prostate $^{13}$, liver ${ }^{14}$, and breast ${ }^{15}$ tumor tissues. Such tumor organoids mimic their original tumors phenotypically and genetically. Due to their similarity to in vivo tumor tissues and their numerous practical applications, researchers have adopted them as novel disease models in the study of cancer pathogenesis.

Here, the procedures for the establishment of tumor organoids from a carcinogen-induced murine invasive urothelial tumor ${ }^{16}$ are laid out. Nbutyl-N-(4-hydroxybutyl) nitrosamine (BBN) is used as a carcinogen to induce invasive urothelial carcinoma in mice ${ }^{17}$ and the tumor organoids, which exhibit the pathological characteristics of mouse muscle-invasive bladder tumors, are established from the BBN-induced murine bladder cancer $^{16}$. The method to genetically manipulate the tumor organoids is illustrated using lentivirus-mediated transduction to develop a model system for studying the molecular basis of the development of bladder cancer. In addition, a method for transplanting organoids orthotopically into a bladder to investigate the role of the native bladder environment in bladder cancer is described. 


\section{Protocol}

All procedures were approved and conducted under the guidelines of the Institutional Animal Care and Use Committee at POSTECH (IACUC number: POSTECH-2019-0055).

\section{In Vitro Culture of Bladder Tumor Organoids}

1. Establish bladder tumor organoids from the murine bladder tumor (Figure 1A)

NOTE: The procedure for generating BBN-induced mouse bladder tumors is outlined in Shin et al. ${ }^{17}$.

1. Provide $0.1 \%$ BBN-containing water in a dark bottle to mouse ad libitum for 6 months. Change BBN-containing water $2 x$ a week. NOTE: A C57BL/6 male mouse with a body weight of approximately $25 \mathrm{~g}$ at $8-10$ weeks of age was used. BBN-containing water can be administered to up to five mice in a single cage.

2. After 6 months, euthanize the mouse using carbon dioxide inhalation and isolate the entire bladder tumor. Transfer it to a $90 \mathrm{~mm}$ Petri dish.

3. Remove non-cancerous parts and necrotic regions using sterile surgical scissors and wash the bladder tumor fragments 2-3 times with cold 1x Dulbecco's phosphate-buffered saline (DPBS). Collect the fragments and transfer them to a new $90 \mathrm{~mm}$ Petri dish.

4. Add $1 \mathrm{~mL}$ of Dulbecco's modified minimum essential medium (DMEM) with $10 \mathrm{mM}$ 4-(2-hydroxyethyl)-1-piperazineethanesulfonic acid (HEPES).

5. Mince the tumor tissue into pieces as small as possible $\left(0.5-1 \mathrm{~mm}^{3}\right)$ using a sterilized razor blade.

6. Add $9 \mathrm{~mL}$ of DMEM with $10 \mathrm{mM} \mathrm{HEPES}, 250 \mu \mathrm{g} / \mathrm{mL}$ collagenase type I, $250 \mu \mathrm{g} / \mathrm{mL}$ collagenase type II, and $250 \mathrm{U} / \mathrm{mL}$ thermolysin. Incubate the minced tumor tissue for $1.5-2 \mathrm{~h}$ on an orbital shaker in an incubator $\left(37^{\circ} \mathrm{C}, 5 \% \mathrm{CO}_{2}\right)$ to dissociate the fragments into the cell suspension. Transfer the cell suspension into a $50 \mathrm{~mL}$ tube.

NOTE: If the size of tumor harvested from the mouse is greater than $1 \mathrm{~cm}^{3}$, treat it with $2 x$ the amount of thermolysin or increase the incubation time.

7. Centrifuge the tube at $400 \times g$ for $5 \mathrm{~min}$ at $4{ }^{\circ} \mathrm{C}$ and aspirate the supernatant.

8. Resuspend the pellet using $5 \mathrm{~mL}$ of ammonium-chloride-potassium (ACK) lysing buffer to lyse any red blood cells. Incubate the tube for 3-5 min at room temperature (RT) until the complete lysis of red blood cells. NOTE: If the red blood cells are not observed, omit the lysing process.

9. Add $20 \mathrm{~mL}$ of DMEM into the tube. Centrifuge the tube at $400 \times \mathrm{g}$ for $5 \mathrm{~min}$ at $4{ }^{\circ} \mathrm{C}$ and aspirate the supernatant.

10. Resuspend the pellet with $1 \mathrm{~mL}$ of $0.25 \%$ Trypsin-EDTA and $10 \mu \mathrm{M} \mathrm{Y}-27632$ dihydrochloride (Y-27632) to dissociate the pellet into single cells. Incubate the tube for $5 \mathrm{~min}$ in a $37^{\circ} \mathrm{C}$ water bath.

NOTE: Observe the tumor under a microscope to confirm complete dissociation into single cells. If chunks of cells persist, pipette the suspension further.

11. Neutralize trypsin using $10 \mathrm{~mL}$ of DMEM with $10 \%$ fetal bovine serum (FBS). Filter the cell suspension through a $100 \mu \mathrm{m}$ cell strainer on a new $50 \mathrm{~mL}$ tube to remove the undigested debris.

12. Centrifuge the tube at $400 \times g$ for $5 \mathrm{~min}$ at $4{ }^{\circ} \mathrm{C}$ and aspirate the supernatant.

13. Coat a well in a 24 well plate using $150 \mu \mathrm{L}$ of ice-cold growth factor reduced basement membrane matrix (Table of Materials) and place the 24 well plate in an incubator $\left(37^{\circ} \mathrm{C}, 5 \% \mathrm{CO}_{2}\right)$ for $30 \mathrm{~min}$ to solidify the basement membrane matrix. NOTE: Thaw and maintain the basement membrane matrix at $4{ }^{\circ} \mathrm{C}$ to prevent solidification before use.

14. Resuspend the pellet using $1 \mathrm{~mL}$ of DMEM and count the cells using a hemocytometer. Transfer $3-4 \times 10^{4}$ tumor cells into a $1.5 \mathrm{~mL}$ microtube on ice.

15. Centrifuge the microtube at $400 \times g$ for $3 \min$ at $4{ }^{\circ} \mathrm{C}$ and carefully discard the supernatant.

16. Resuspend the cells with $500 \mu \mathrm{L}$ of prewarmed organoid medium (Table 1) and $10 \mu \mathrm{M} \mathrm{Y}-27632$ and transfer them into the coated well. Place the 24 well plate in an incubator $\left(37^{\circ} \mathrm{C}, 5 \% \mathrm{CO}_{2}\right)$

17. Extra bladder tumor cells can be stocked with $1 \mathrm{~mL}$ of DMEM containing $10 \% \mathrm{FBS}, 1 \%$ penicillin/streptomycin, and $10 \%$ dimethyl sulfoxide (DMSO) in $1.5 \mathrm{~mL}$ cryovials. Place them in a cryovial freezing container and transfer the container to a $-80^{\circ} \mathrm{C}$ freezer. After storage in the freezer overnight, transfer the cryovials into liquid nitrogen for long-term storage.

18. Change the medium every 2 days using $500 \mu \mathrm{L}$ of prewarmed organoid medium (Figure 1B).

2. Subculture bladder tumor organoids.

NOTE: Passage of bladder tumor organoids when they reach $100-150 \mu \mathrm{m}$ in diameter is recommended.

1. Add $500 \mu \mathrm{L}$ of collagenase/dispase to the organoid medium in the 24 well plate with tumor organoids. Pipette up and down the basement membrane matrix and the medium. Incubate for $20 \mathrm{~min}$ at $37^{\circ} \mathrm{C}$ and harvest the cells into a $15 \mathrm{~mL}$ tube.

NOTE: Examine the organoids isolated from the basement membrane matrix under a microscope. If the organoids are not detached from the basement membrane matrix, increase the incubation time or pipette more times.

2. Add $5 \mathrm{~mL}$ of prewarmed DMEM, centrifuge the tube at $400 \times \mathrm{g}$ for $3 \mathrm{~min}$ at $4{ }^{\circ} \mathrm{C}$, and aspirate the supernatant.

3. Resuspend the pellet using $1 \mathrm{~mL}$ of prewarmed $0.25 \%$ trypsin-EDTA and $10 \mu \mathrm{M} \mathrm{Y}-27632$. Incubate for 5 min in a $37^{\circ} \mathrm{C}$ water bath. Vigorously pipette the cells up and down and neutralize the trypsin using $5 \mathrm{~mL}$ of DMEM with $10 \% \mathrm{FBS}$.

4. Centrifuge the tube at $400 \times g$ for 3 min at $4{ }^{\circ} \mathrm{C}$ and aspirate the supernatant.

5. Resuspend the pellet using $1 \mathrm{~mL}$ of prewarmed organoid medium and count the number of single tumor cells.

6. Repeat steps 1.1.14-1.1.18. 


\section{Genetic Manipulation of Bladder Tumor Organoids Using Lentivirus-mediated Transduction (Figure 2A)}

1. Produce the GFP-expressing lentiviral particles.

1. On day 0 , plate HEK 293 T cells at a density of $5-6 \times 10^{6}$ cells per $10 \mathrm{~cm}$ cell culture plate in cell line culture medium (i.e., DMEM with $10 \%$ FBS and $1 \%$ penicillin/streptomycin).

2. On day 1 , prepare the DNA transfection solution including transfer plasmid containing GFP $(8 \mu \mathrm{g})$, lentiviral packaging plasmid (10 $\mu \mathrm{g}$ of pCMVR 8.74 and $3 \mu \mathrm{g}$ of pMD2.G), and $1 \mathrm{~mL}$ of reduced serum medium (Table of Materials).

3. Add $3 \mu \mathrm{L}$ of transfection reagent (Table of Materials) per $1 \mu \mathrm{g}$ of total plasmid according to the manufacturer's instructions and mix gently by pipetting. Incubate for 20 min at RT and add $9 \mathrm{~mL}$ of DMEM with $10 \%$ FBS.

4. Aspirate the culture medium in the cell culture plate with HEK 293T cells. Carefully transfer $10 \mathrm{~mL}$ of the DNA transfection solution onto the HEK 293T cells and incubate in a cell culture incubator at $37^{\circ} \mathrm{C}$.

5. On day 3, observe the cells under a fluorescence microscope (excitation at $488 \mathrm{~nm}$ and emission at $512 \mathrm{~nm}$ ) to determine the transfection efficiency. Almost $90 \%-100 \%$ of the cells in the entire cell population should express GFP.

6. Collect the supernatant (containing the virus) and filtrate the supernatant with a $0.45 \mu \mathrm{m}$ polyethersulfone (PES) filter. NOTE: Use a low protein-binding filter such as a PES filter.

7. To concentrate the virus, centrifuge the virus supernatant at $98,768 \times g$ in an ultracentrifuge for $2 \mathrm{~h}$ at $4{ }^{\circ} \mathrm{C}$ in a swinging bucket rotor (Table of Materials) and carefully discard the supernatant.

8. Resuspend the pellet in $2.5 \mathrm{~mL}$ of cold organoid medium.

9. For long-term storage, aliquot $250 \mu \mathrm{L}$ of lentiviral medium into cryogenic vials and snap-freeze them using liquid nitrogen. Store the frozen viral stocks in a $-80^{\circ} \mathrm{C}$ freezer.

2. Perform lentivirus-mediated transduction of the bladder tumor organoids

1. On day 2, split the tumor organoids as described above (step 1.2) $12 \mathrm{~h}$ before the lentivirus-mediated transduction.

2. On day 3 , quickly thaw an aliquot (step 2.1.9) containing virus in a $37^{\circ} \mathrm{C}$ water bath and add the $250 \mu \mathrm{L}$ of organoid medium with 10 $\mu \mathrm{M} \mathrm{Y}-27632$ and $8 \mu \mathrm{g} / \mathrm{mL}$ hexadimethrine bromide.

3. Replace the organoid medium in the 24 well plate with tumor organoids by $500 \mu \mathrm{L}$ of virus-containing medium and incubate for $12-16 \mathrm{~h}$ in an incubator $\left(37^{\circ} \mathrm{C}, 5 \% \mathrm{CO}_{2}\right)$.

4. On day 4 , change the medium with $500 \mu \mathrm{L}$ of fresh organoid medium.

NOTE: After $12-16 \mathrm{~h}$ of incubation, the medium should be changed, because the medium containing lentivirus and hexadimethrine bromide is cytotoxic.

5. On day 6, monitor the GFP signal from the tumor organoids 3 days after transduction under a fluorescence microscope (Figure 2B)

6. On day 10 , passage and stock the organoids 7 days after transduction as described in step 1.2, to maintain the genetically modified tumor organoid lines.

\section{Orthotopic Transplantation of Bladder Organoid (Figure 3A)}

1. Prepare the bladder tumor organoids for orthotopic transplantation.

1. Before transplantation, culture the bladder tumor organoids for 5-7 days, as described above (step 1.2).

2. Add $500 \mu \mathrm{L}$ of collagenase/dispase to organoid medium in a 24 well plate with the tumor organoids. Pipette up and down the basement membrane matrix and medium. Incubate for $20 \mathrm{~min}$ at $37^{\circ} \mathrm{C}$ and collect the cells into a $15 \mathrm{~mL}$ tube.

3. Add $5 \mathrm{~mL}$ of prewarmed DMEM, centrifuge the tube at $400 \times g$ for $3 \mathrm{~min}$ at $4{ }^{\circ} \mathrm{C}$, and aspirate the supernatant.

4. Resuspend the pellet with $1 \mathrm{~mL}$ of DMEM and transfer the solution into a $90 \mathrm{~mm}$ Petri dish.

5. Under a microscope, pick up the 10-100 tumor organoids by using a p200 micropipette and collect them into a microtube on ice.

6. Centrifuge the tube at $400 \times g$ for $3 \mathrm{~min}$ at $4{ }^{\circ} \mathrm{C}$ and carefully discard the supernatant.

7. Maintain the cell pellet on ice until the mice are ready for surgery.

2. Submucosal bladder wall transplantation

NOTE: This procedure is modified from the protocol published by Fu et $\mathrm{al}^{18}$.

1. Prepare an 8 to 10 week-old male nude mouse $(\mathrm{CAnN}$.Cg-Foxn1 $\mathrm{nu} / \mathrm{Crl})$ at least 1 week before the experiment to allow it to acclimate to a new environment. Inject enrofloxacin $(5 \mathrm{mg} / \mathrm{kg}$ ) subcutaneously $24 \mathrm{~h}$ before surgery.

2. Clean the bench surface by soap and water. Autoclave the surgical instruments prior to the surgical procedure and perform surgery using sterile instruments.

3. Keep the $29 \mathrm{G}$ insulin syringe, pipette tips, and basement membrane matrix on ice. Administer ketoprofen (5 mg/kg) subcutaneously before administration of anesthesia.

4. Anesthetize the mouse with $4 \%$ isoflurane in an induction chamber. Once general anesthesia achieved, lay the mouse in a supine position and maintain anesthesia by mask inhalation of $2 \%$ vaporized isoflurane.

NOTE: If the anesthetization time is over $30 \mathrm{~min}$, apply eye ointment to both eyes using a cotton swab to avoid corneal drying.

5. Apply povidone-iodine with a sterile gauze and wipe it down with $70 \%$ ethanol. Repeat $3 x$ with a new gauze or a cotton swab each time.

6. Cover the anus and the surgical field using disposable, sterile surgical drapes. Inject sustained release buprenorphine (1.0 mg/kg) once before skin incision.

7. Using a dissecting microscope for magnification, make a small transverse incision (smaller than $1.5 \mathrm{~cm}$ ) in the skin and muscular wall of the lower midline abdomen with sterile surgical scissors. Expose the bladder from the abdominal cavity and support it with salinesoaked cotton swabs.

NOTE: If the bladder is full of urine, gently press the bladder to decompress it slightly. 
8. Resuspend the organoid pellets (step 3.1.7) in $80 \mu \mathrm{L}$ of organoid medium containing $50 \%$ high-concentration basement membrane matrix (Table of Materials).

9. Inject the organoid suspension into the anterior aspect of the bladder dome using the $29 \mathrm{G}$ insulin syringe under a dissecting microscope.

10. Close the inner layer of the abdominal wall with antibacterial absorbable suture and then close the outer layer with 4-0 nylon suture. Disinfect the surgical site with povidone-iodine and $70 \%$ ethanol.

11. Allow the mouse to recover under an infrared irradiator 10-15 min. Monitor the mouse until it regains consciousness and motility.

12. One day after surgery, check the general condition of the mouse and anastomotic leakage. Administer ketoprofen $(5 \mathrm{mg} / \mathrm{kg}) \mathrm{once}$ daily for 3 days post-operatively and treat enrofloxacin $(5 \mathrm{mg} / \mathrm{kg})$ once daily for 10 days post-operatively.

13. When incision site has healed (10-14 days after surgery), remove the sutures. Monitor the growth of the mouse bladder tumor for $2-3$ weeks after the tumor organoid injection.

14. If bladder tumor growth is observed, euthanize the mouse using carbon dioxide inhalation, and harvest the entire bladder tumor. Wash it using cold DPBS (Figure 3B) ${ }^{16}$.

15. To analyze the bladder tumor histology, stain the paraffin-embedded section of the tissue using hematoxylin and eosin $(\mathrm{H}$ and $\mathrm{E})$ staining (Figure 3B) ${ }^{16}$.

\section{Representative Results}

In vitro culture of mouse bladder tumor organoids

The number of tumor cells dissociated from an $\sim 1 \mathrm{~cm}^{3}$ BBN-induced tumor is at least $4 \times 10^{5}$ cells. When the cells are initially seeded in the basement membrane matrix, non-cancerous cells and debris may be observed. Debris was gradually diluted out by continuing the subculture. Figure 1B shows images of the cultured organoids at different time points. If the tumor cells do not form tumor organoids, the cells are potentially dead during the dissociation step. In such a case, dissociation procedures including incubation time with the enzyme need to be adjusted to increase cell viability.

\section{Expression of GFP in bladder tumor organoids using lentivirus-mediated genetic manipulation}

Bladder tumor organoids exhibited strong GFP signals with successful lentiviral infection (Figure 2B). After concentration, a total of $250 \mu \mathrm{L}$ of virus-containing media was enough to infect $3 \times 10^{4}$ single tumor cells on the basement membrane matrix, maintaining $90 \%-100 \%$ infection efficiency. GFP signals could be detected from the bladder tumor organoids 3 days after lentiviral transduction. If the fluorescence signals are low, the efficiency of viral infection is potentially low. This can be due to numerous factors, such as low viral titer, and the procedures need to be adjusted accordingly.

\section{Orthotopic transplantation of bladder tumor organoids}

A bladder tumor allograft obtained from BBN-induced bladder tumor organoids is presented in Figure $3 \mathbf{B}^{16}$. Bladder tumor allografts were harvested 3 weeks after orthotopic transplantation. The histology of the transplanted bladder tumor was analyzed using $\mathrm{H}$ and $\mathrm{E}$ staining. Orthotopic transplants of tumor organoids can grow as bladder tumors for 2-3 weeks.

A

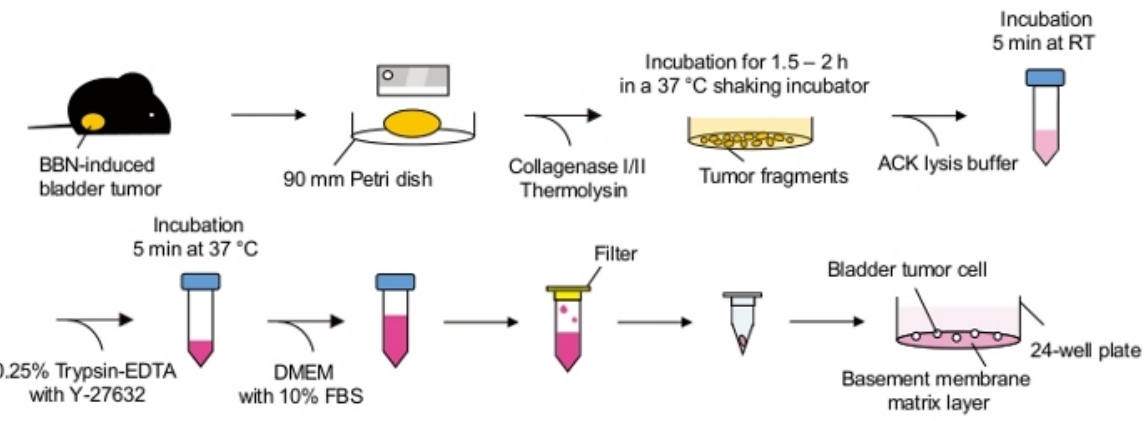

B

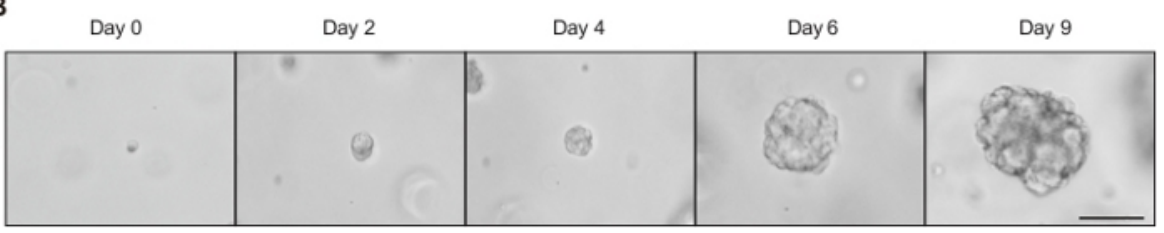

Figure 1: In vitro culture of mouse bladder tumor organoids. (A) Schematic diagram for the establishment of mouse bladder tumor organoids. (B) Representative images for the culture of bladder tumor organoids at different time points. Mouse bladder tumor organoids were established and cultured over 9 days. Scale bar $=100 \mu \mathrm{m}$. Please click here to view a larger version of this figure. 
A

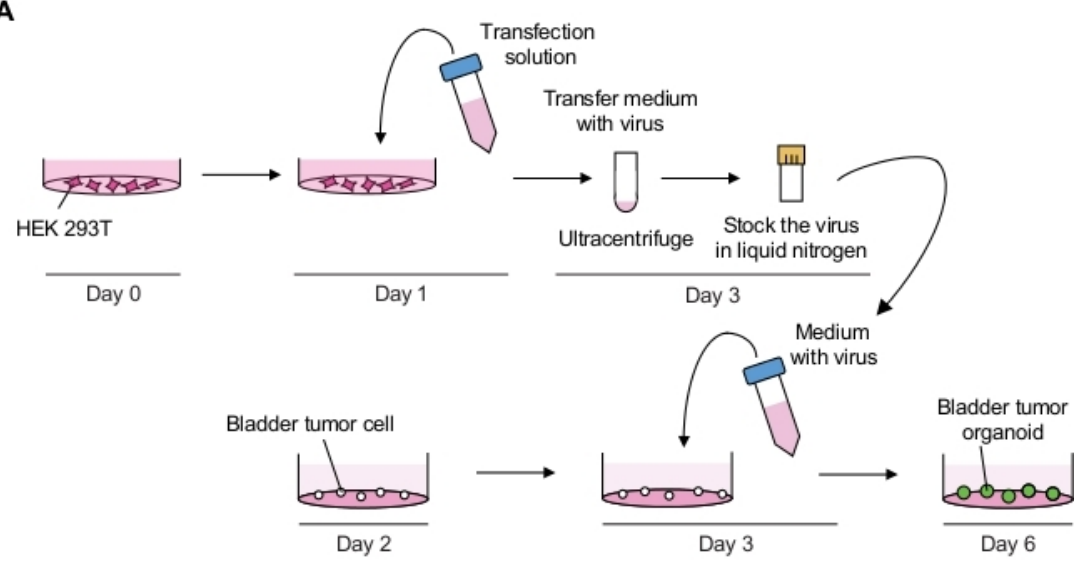

B

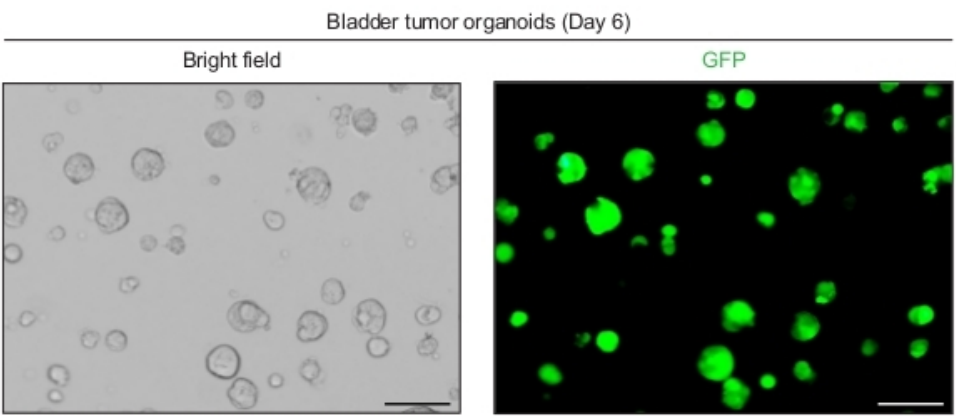

Figure 2: Expression of GFP in bladder tumor organoids using lentivirus-mediated genetic manipulation. (A) Schematic diagram of lentiviral transfection and transduction of bladder tumor organoids. (B) Representative images of bladder tumor organoids expressing GFP. Scale bars $=100 \mu \mathrm{m}$. Please click here to view a larger version of this figure.

A

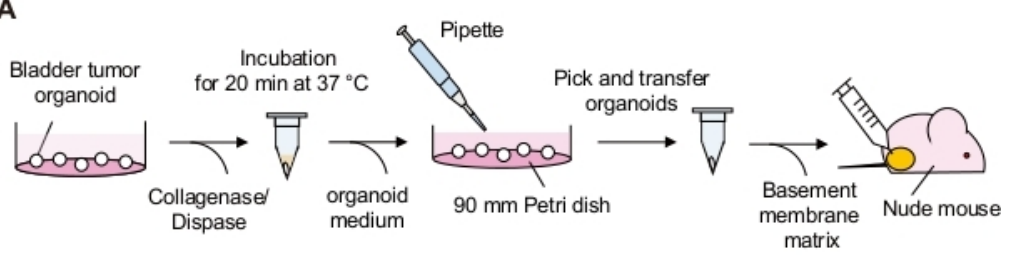

B

Orthotopic transplantation of tumor organoids

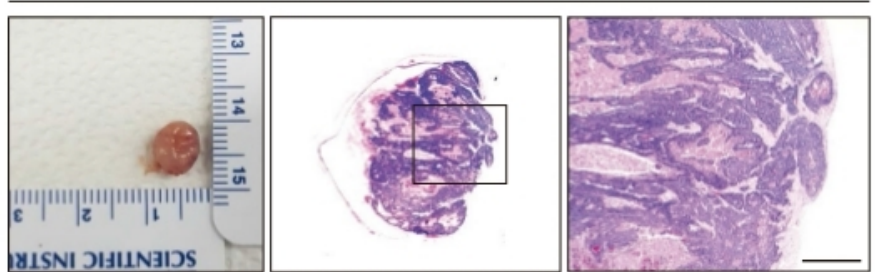

Figure 3: Orthotopic transplantation of bladder tumor organoids. (A) Schematic diagram of orthotopic transplantation of bladder tumor organoids to a nude mouse. (B) Representative images of bladders and $\mathrm{H}$ and $\mathrm{E}$ stained sections from mice orthotopically transplanted with bladder tumor organoids. Magnified views of the boxed regions in the middle panels are shown in the left panels. Scale bar $=500 \mu \mathrm{m}$. This figure was reproduced from Figure 1-Figure Supplement 1, Kim et al. ${ }^{16}$, published under the Creative Commons Attribution 4.0 International Public License (CC BY 4.0; https://creativecommons.org/licenses/by/4.0/). Please click here to view a larger version of this figure. 


\begin{tabular}{|l|l|}
\hline \multicolumn{2}{|l|}{ Mouse bladder tumor organoids medium } \\
\hline Advanced DMEM/F-12 (Basic medium) & $10 \mathrm{mM} \mathrm{HEPES}(\mathrm{pH} 7.4)$ \\
\hline $10 \mathrm{mM}$ Nicotinamide & $0.5 x$ Serum-free supplement \\
\hline $2 \mathrm{mM}$ L-alanyl-L-glutamine dipeptide & $1 \%$ Penicillin/Streptomycin \\
\hline $1 \mathrm{mM} \mathrm{N}$-acetyl-L-cysteine & $50 \mathrm{ng} / \mathrm{mL}$ Murine epidermal growth factor \\
\hline $1 \mu \mathrm{M} \mathrm{A} \mathrm{83-01}$ & \\
\hline
\end{tabular}

Table 1: Composition of bladder tumor organoid medium.

\section{Discussion}

This protocol describes the experimental procedures to culture and maintain bladder tumor organoids derived from carcinogen-induced murine bladder tumors.

In this protocol, there are several experimental steps in which the procedures might need some troubleshooting. First, the number of tumor cells that are initially seeded is a critical factor because low numbers of tumor cells in culture $\left(<2 \times 10^{4}\right.$ cells $)$ mostly lead to cell death due to lack of interactions among tumor cells. In contrast, beginning with too many cells ( $>5 \times 10^{4}$ cells) at seeding leads to overcrowded organoids, resulting in difficulty when handling cultures with poor growth of each organoid. It is strongly suggested that multiple plates with different numbers of cells be established at the beginning to optimize the experimental conditions. Identifying the right number of initial tumor cells is crucial to achieve the highest cell viability and to establish successful bladder tumor organoids. Also, in long-term culture of over 2 weeks without passaging, most tumor organoids stop growing, potentially due to inadequate supply of nutrients at the center of the organoids and the depletion of growth factor in the basement membrane matrix. Therefore, subculturing organoids in a timely manner is a critical step to maintain tumor organoid culture.

Second, the production of high-titer lentiviral particles is critical for the efficient genetic manipulation of tumor organoids. To troubleshoot virus titer-related issues, it is strongly suggested that the virus titers be determined before viral transduction every time because lentiviral constructs tend to produce viral particles with varying efficiency. If tumor organoids exhibit low viability following viral infection, it is likely that the viral titers are potentially too high. It is strongly suggested to use lower amount of virus in this case. Third, during orthotopic transplantation of BBN-induced bladder tumor organoids, it is critical to maintain the integrity of the bladder wall. In case that the injection reaches the lumen of the bladder by penetrating the bladder wall layer, the experiment should be terminated and discarded. If possible, the monitoring of bladder tumor growth using an ultrasound imaging system is recommended.

One limitation of the current techniques is the absence of the tumor microenvironment or stroma in these organoids. To overcome this issue, it is strongly suggested that the orthotopic transplantation of tumor organoids use an in vivo system to mimic the native tumor microenvironment. In the future, it will be necessary to develop 3D in vitro organoid systems that are composed of tumor organoids with other components of tumor stroma.

One of the major implications of our technique is that, in orthotopic transplantation of tumor organoids, only 10 bladder tumor organoids can induce tumor growth in the bladder. Compared to the conventional tumor transplantation experiments that require $5 \times 10^{5}-1 \times 10^{6}$ single bladder tumor cells, our methods are much more efficient and robust. Another significant difference is that the organoids can be diversely manipulated using various lentiviral vectors, such as lentiviral constructs containing short-hairpin RNA, the CRISPR-Cas9 system, or genes of interest. These would be powerful tools to add to current organoid technology. Overall, the experimental approaches presented here can facilitate the establishment of in vitro tumor models that can improve our understanding of the pathogenesis of bladder cancer rather than using 2D bladder cancer cell lines.

This method was able to establish bladder tumor organoids derived from a carcinogen-induced murine bladder tumor. The article provides a description of the lentivirus-mediated experimental procedures through which the genetic modifications are introduced and stably maintained in bladder tumor organoids. In addition, a procedure for orthotopic transplantation of tumor organoids is included. In combination with current in vivo cancer models, this technique will be a useful tool to study the molecular basis of bladder tumorigenesis.

\section{Disclosures}

The authors declare no competing financial interests.

\section{Acknowledgments}

This research was supported by grants from the National Research Foundation of Korea to K.S: NRF-2017R1A2B4006043,

NRF-2017M3C7A1047875, NRF-2017R1A5A1015366, Creative Economy Leading Technology Development Programme (SF317001A), POSCO (2018Y060) and the BK21 Plus Research Fellowship.

\section{References}

1. Sanli, O. et al. Bladder cancer. Nature Reviews Disease Primers. 3, 17022 (2017).

2. Stenzl, A. et al. Treatment of muscle-invasive and metastatic bladder cancer: update of the EAU guidelines. European Urology. 59 (6), 10091018 (2011). 
3. Barretina, J. et al. The Cancer Cell Line Encyclopedia enables predictive modelling of anticancer drug sensitivity. Nature. 483 (7391), 603 (2012).

4. Caponigro, G., Sellers, W. R. Advances in the preclinical testing of cancer therapeutic hypotheses. Nature Reviews Drug Discovery. 10 (3), 179 (2011).

5. Drost, J., Clevers, H. Organoids in cancer research. Nature Reviews Cancer. 18 (7), 407 (2018).

6. Fatehullah, A., Tan, S. H., Barker, N. Organoids as an in vitro model of human development and disease. Nature Cell Biology. 18 (3), 246 (2016).

7. Pauli, C. et al. Personalized in vitro and in vivo cancer models to guide precision medicine. Cancer discovery. 7 (5), $462-477$ (2017).

8. Sato, T. et al. Long-term expansion of epithelial organoids from human colon, adenoma, adenocarcinoma, and Barrett's epithelium. Gastroenterology. 141 (5), 1762-1772 (2011).

9. van de Wetering, M. et al. Prospective derivation of a living organoid biobank of colorectal cancer patients. Cell. 161 (4), $933-945$ (2015).

10. Lee, S. H. et al. Tumor evolution and drug response in patient-derived organoid models of bladder cancer. Cell. 173 (2), 515-528. e517 (2018).

11. Boj, S. F. et al. Organoid models of human and mouse ductal pancreatic cancer. Cell. 160 (1-2), 324-338 (2015).

12. Huang, L. et al. Ductal pancreatic cancer modeling and drug screening using human pluripotent stem cell-and patient-derived tumor organoids. Nature Medicine. 21 (11), 1364 (2015).

13. Gao, D. et al. Organoid cultures derived from patients with advanced prostate cancer. Cell. 159 (1), 176-187 (2014).

14. Broutier, L. et al. Human primary liver cancer-derived organoid cultures for disease modeling and drug screening. Nature Medicine. 23 (12), 1424 (2017).

15. Sachs, N. et al. A living biobank of breast cancer organoids captures disease heterogeneity. Cell. 172 (1-2), 373-386. e310 (2018).

16. Kim, S. et al. Epigenetic regulation of mammalian Hedgehog signaling to the stroma determines the molecular subtype of bladder cancer. eLife. 8, e43024 (2019).

17. Shin, K. et al. Cellular origin of bladder neoplasia and tissue dynamics of its progression to invasive carcinoma. Nature Cell Biology. 16 (5), 469 (2014).

18. Fu, C. L., Apelo, C. A., Torres, B., Thai, K. H., Hsieh, M. H. Mouse bladder wall injection. JoVE (Journal of Visualized Experiments). 12 (53), e2523 (2011). 